\title{
Flavonoid-rich-berry-extract treatment decreases the expression of DMT1 and functionally-similar metal transporter genes in human intestinal Caco-2 cells
}

\author{
F. Alzaid ${ }^{1}$, K. Pourvali ${ }^{1}$, C. I. Lin $^{1}$, M. Arno ${ }^{1}$, E. Aldecoa-Otalora Astarloa ${ }^{1}$, P. A. Sharp ${ }^{1}$, \\ C. Hogstrand ${ }^{1}$, P. W. Emery ${ }^{1}$, D. Bagchi ${ }^{2}$, V. R. Preedy ${ }^{1}$ and H. Wiseman ${ }^{1}$ \\ ${ }^{1}$ Nutritional Sciences Division, King's College London, Franklin-Wilkins Building, 150 Stamford Street, London SE1 9NH, \\ $U K$ and ${ }^{2}$ Department of Pharmacological and Pharmaceutical Sciences, University of Houston College of Pharmacy, \\ Houston, Texas 77204, USA
}

Berries are a rich dietary source of bioactive polyphenols including flavonoids such as anthocyanins ${ }^{(1)}$. The ability of flavonoids to chelate divalent metal ions may contribute to their antioxidant action. Dietary polyphenols are known to impair Fe absorption. They can reduce non-haem-Fe transport across Caco-2 cell monolayers ${ }^{(2)}$. However, little is known about their influence on the expression of the genes involved. The present study investigated the influence of berry polyphenols on the expression of metal transporter and related genes.

Human intestinal Caco- 2 cells were cultured for $21 \mathrm{~d}$ and were then treated for $16 \mathrm{~h}$ with an anthocyanin-rich berry extract (OptiBerry; InterHealth Nutraceuticals, Benicia, CA, USA) at a final concentration of $0.5 \%$ (w/v). Subsequently, mRNA was extracted, pooled and used for the microarray analysis using Affymetrix gene chip HG-U133A (Affymetrix Inc., Santa Clara, CA, USA) and for quantitative RT-PCR. Microarray data were analysed using $\operatorname{GCOS}^{\mathrm{TM}}$ software (Affymetrix Inc.) and the Database for Annotation, Visualisation and Integrated Discovery (version 4; Laboratory of Immunopathogenesis and Bioinformatics, National Cancer Institute at Frederick, Frederick, MD, USA). A cut-off of 2-fold change and $P \leq 0.05$ was applied.

DMT1 (divalent metal transporter; cellular Fe absorption), ZIP2 (Zn uptake transporter), ZNT10 (Zn efflux transporter), ATP7B (Cu efflux transporter), MRS2 (Mg uptake transporter) and HFE (regulation of Fe absorption) were identified as a novel group of metal transporter and related genes affected by the treatment. Functional similarity of these genes to DMT1 was determined (Fig. 1). Expression of DMT1, ZNT10, ZIP2, HFE, ATP7B and MRS2 was decreased by the treatment (Fig. 2) and the microarray data was validated by quantitative RT-PCR using the $18 \mathrm{~S}$ amplicon as a housekeeping gene (mean fold decreases 1.70 (SD 0.73), 1.74 (SD 0.56 ), 1.74 (SD 0.82 ), 2.43 (SD 0.72), 1.11 (SD 0.36) and 1.11 (SD 0.36) respectively).

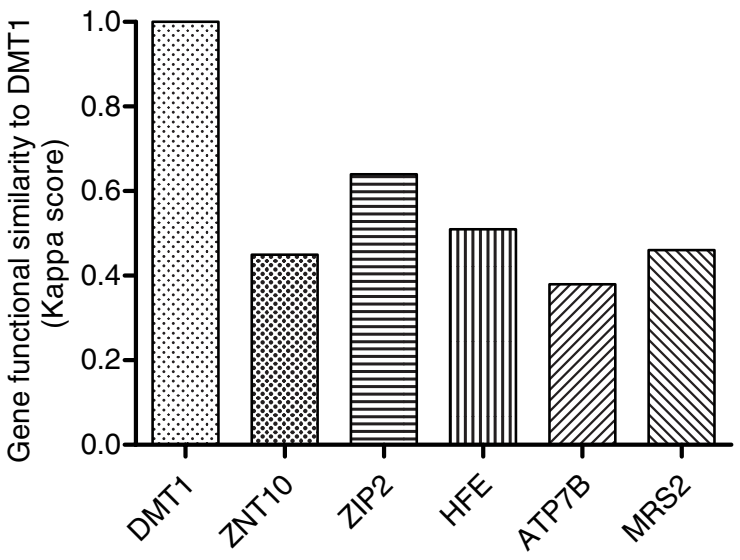

Metal transporter and related genes

Fig. 1. Functional similarity to DMT1 of metal transporter and related genes.

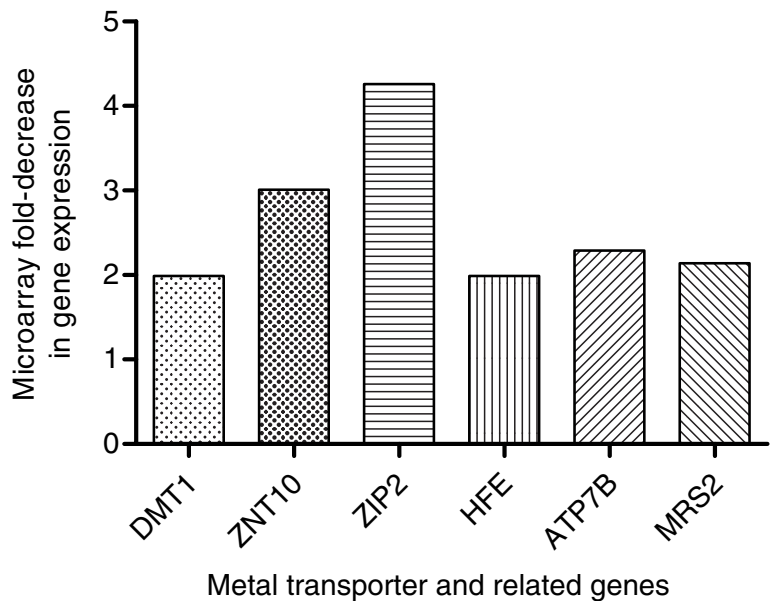

Fig. 2. Microarray fold-decrease in expression of metal transporter and related genes.

Studies are in progress to investigate the biological relevance of the observed effects in relation to berry consumption and the absorption of dietary $\mathrm{Fe}$ and $\mathrm{Zn}, \mathrm{Cu}$ and $\mathrm{Mg}$.

1. Zafra-Stone S, Yasmin T, Bagchi M et al. (2007) Mol Nutr Food Res 51, 675-683.

2. Kim EY, Ham SK, Shigenaga MK et al. (2008) J Nutr 138, 1647-1651. 